Supporting Information

\title{
Urease-Powered Polydopamine Nanomotors for Intravesical Therapy of Bladder Diseases
}

Hyunsik Choi, Seong Hwi Cho and Sei Kwang Hahn*

Department of Materials Science and Engineering, Pohang University of Science and

Technology (POSTECH), 77 Cheongam-ro, Nam-gu, Pohang, Gyeongbuk 37673, Korea.

* CORRESPONDING AUTHOR FOOTNOTE

Tel.: +82 54279 2159; Fax: +82 54279 2399; E-mail address: skhanb@postech.ac.kr (S. K. Hahn) 


\section{Supporting Figures}

A

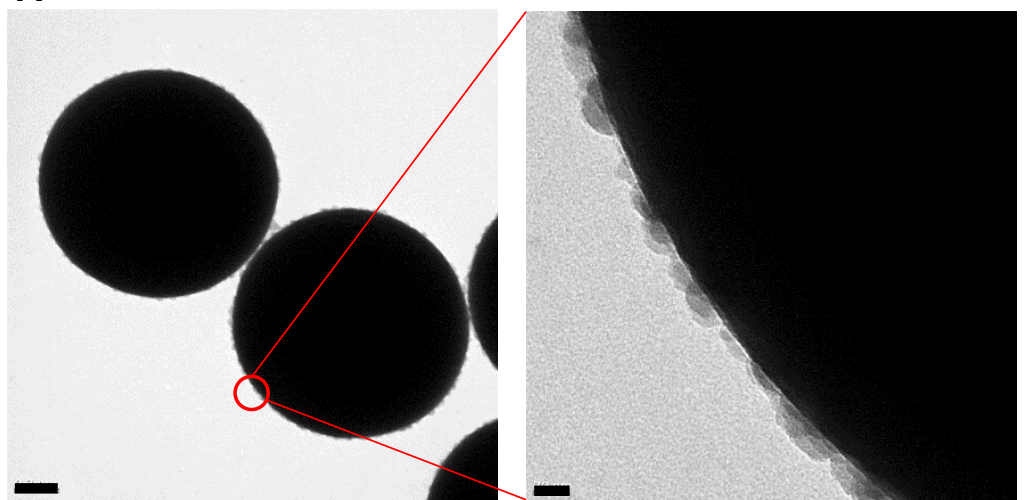

B

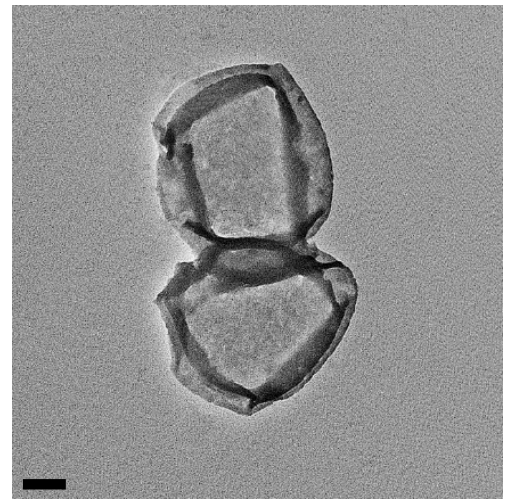

Figure S1. (A) TEM images of PDA@SiNP (left, scale bar=200 nm) and the corresponding magnified images (right, scale bar $=20 \mathrm{~nm}$ ). (B) TEM image of PDA NC (scale bar $=200 \mathrm{~nm})$. 


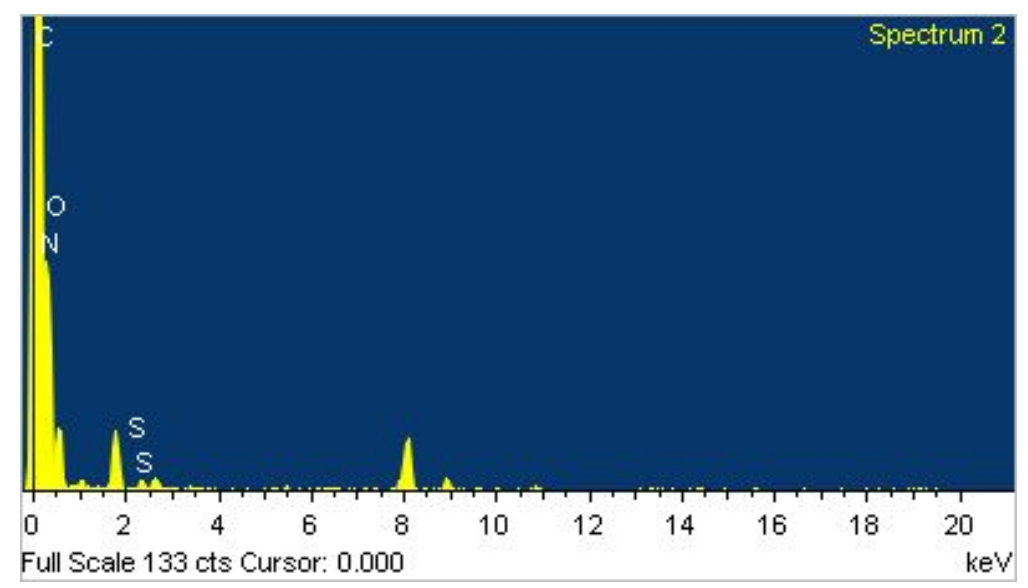

\begin{tabular}{|c|c|c|}
\hline Element & Weight $\%$ & Atomic $\%$ \\
\hline $\mathrm{C}$ & 68.02 & 73.03 \\
\hline $\mathrm{N}$ & 13.93 & 12.83 \\
\hline $\mathrm{O}$ & 17.03 & 13.73 \\
\hline $\mathrm{S}$ & 1.01 & 0.41 \\
\hline & & \\
\hline Totals & 100.00 & \\
\hline
\end{tabular}

Figure S2. EDX spectrum and the element (\%) of urease-powered nanomotors. 


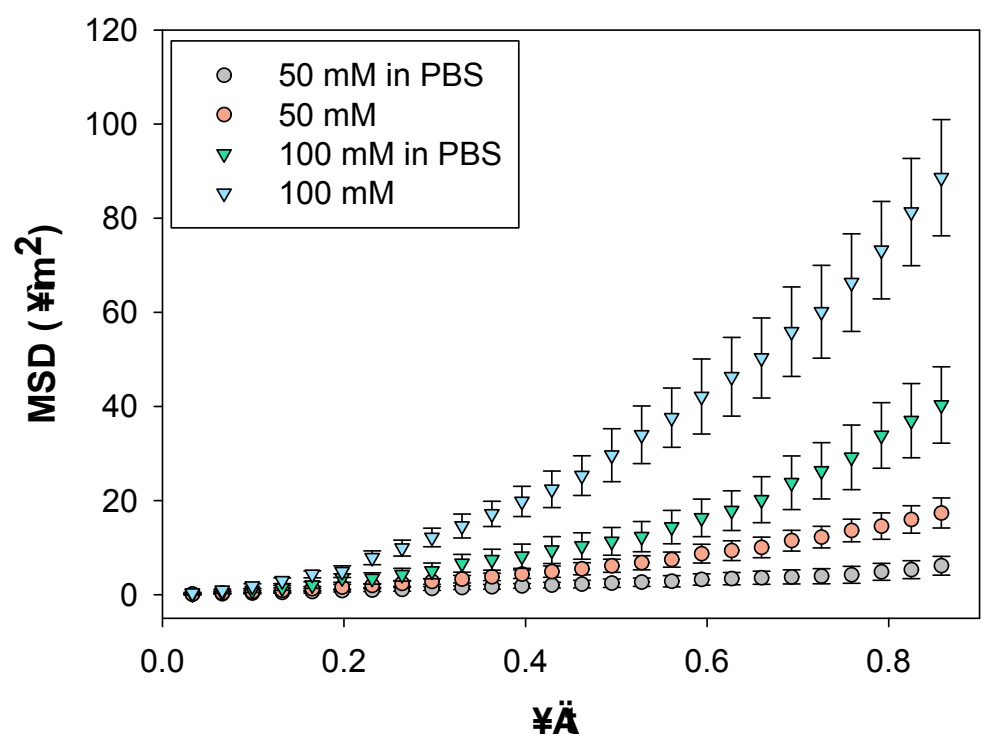

Figure S3. The mean square displacement (MSD) in DI water or PBS with increasing lag time, analyzed by tracking 20 particles in $\mathrm{X}$ and $\mathrm{Y}$ coordinates. 
A

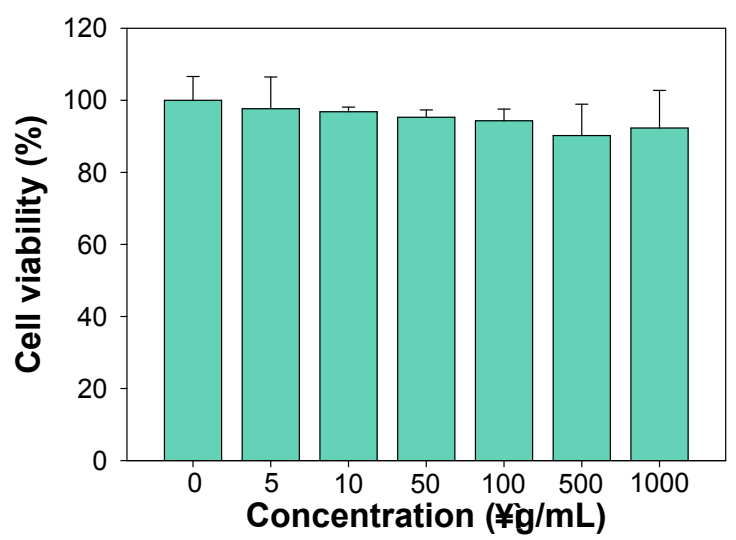

B

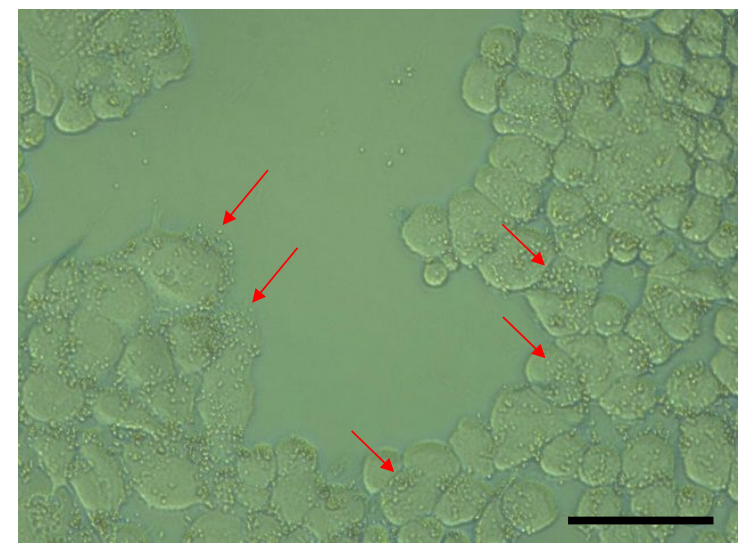

Figure S4. (A) The biocompatibility of urease-powered nanomotors by MTT assay after $24 \mathrm{~h}$ incubation with human bladder cancer cells (RT4) and (B) the corresponding optical image (scale bar $=100 \mu \mathrm{m}$ ). Red arrows indicate the urease-powered nanomotors attached to the cancer cells. 


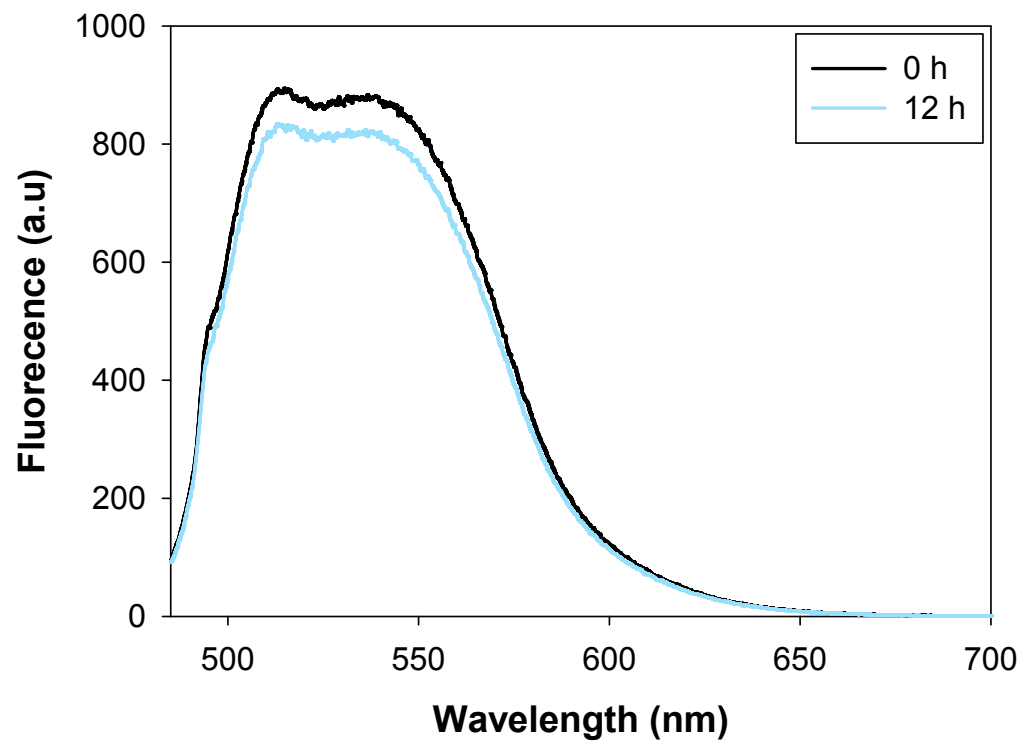

Figure S5. Fluorescence intensity of FITC-labelled nanomotors with increasing wavelength in PBS for $12 \mathrm{~h}$. 


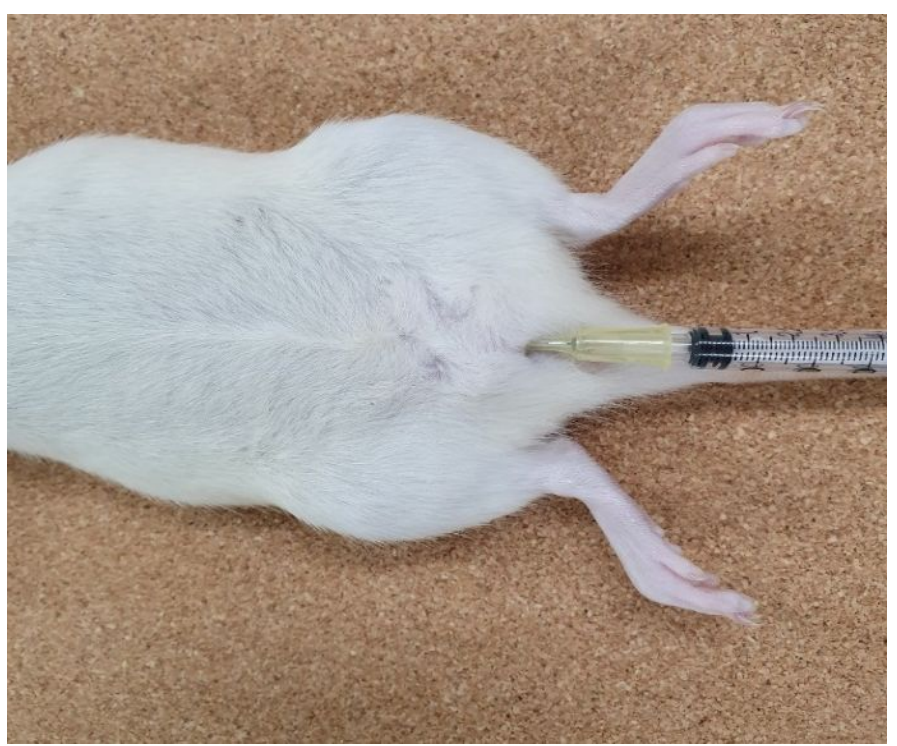

Figure S6. A photo-image for the intravesical injection of urease-powered nanomotors to an SD rat via a catheter. 